\title{
Epidemiological Assessment of GI BioFire Negative Tests at Parkview Health
}

\author{
Brycen Ratcliffe B.S. ${ }^{1}$, Candace Rodgers R.N. ${ }^{2}$, R. Scott Stienecker M.D. ${ }^{2}$ \\ ${ }^{1}$ Indiana University School of Medicine, ${ }^{2}$ Parkview Health Infection Prevention \& \\ Control Department
}

\section{Background and Hypothesis:}

With advances in multiplex PCR testing, many gastrointestinal pathogens can be identified in about an hour and provide $23 \%$ increased yield of pathogens (Beatty et al., 2016). At our facility, $60.4 \%$ of these samples return with no microorganisms found. This study implemented diagnostic stewardship principles by evaluating the negative GI BioFire multiplex results and ordering patterns of providers at Parkview Health.

\section{Design:}

This retrospective chart review of 300 negative GI BioFire results from 2018 pulled demographics and records from Parkview LIS and EPIC software. Inclusion Criteria: negative gastrointestinal BioFire results. Exclusion Criteria: positive results, negative results outside the study period, any patient data not admitted at Parkview Health.

\section{Results:}

$57.0 \%$ of patients had diarrhea-associated co-morbidities. $14.3 \%$ had redundant tests. $51.0 \%$ were administered laxatives, $72.3 \%$ were administered antibiotics. $73.4 \%$ did not follow ACG guidelines stating diarrhea must have persisted longer than seven days. $79.3 \%$ had laxatives concurrently administered or did not follow ACG guidelines. 19.7\% had endoscopic procedures within eight weeks of assay testing. $14.4 \%$ of providers documented a non-C Diff suspicion when ordering assay. $18.9 \%$ had non-reported diarrhea consistency. $45.9 \%$ were hospitalists, $5.5 \%$ were surgeons, and $17.9 \%$ were infectious disease or gastroenterologists. $78.3 \%$ had at least one C-Diff diarrhea risk factor.

\section{Conclusion and Potential Impact:}

This data suggests using appropriate algorithms for GI BioFire and utilizing C-Diff antigen testing is more cost effective and should be used before GI BioFire for those with risk factors. Following ACG guidelines, and deferring those administered laxatives would remove over three-quarters of negative panels. Documentation of stool consistency, volume and suspicious etiologies are essential to furthering diagnostic stewardship. These recommendations will save approximately $\$ 1,036,399$ after consideration for C-Diff antigen testing replacement per year for Parkview Health. 